TEMAS DE ACTUALIDAD

Rev Chil Salud Pública 2019,

Vol 23(2): 161-165

\section{EL DESAFÍO DEL FINANCIAMIENTO DEL SISTEMA DE SALUD CHILENO}

THE CHALLENGE OF HEALTH SYSTEM FINANCING IN CHILE
Uno de los temas centrales que ha emergido con fuerza desde la demanda ciudadana en el contexto del estallido social de octubre de 2019 es salud. En rigor, como tema, las inequidades en salud y los problemas de nuestro sistema sanitario no son nuevos para la academia de la salud pública ni para los ciudadanos. En efecto, desde la recuperación de la democracia, salud ha estado regularmente entre las primeras prioridades para las personas en las diversas encuestas que se han venido desarrollando. Por otra parte, desde años la academia también ha hecho esfuerzos por visibilizar las inequidades en salud ${ }^{1-3}$ y también por hacer propuestas de cambio al sistema sanitario ${ }^{4-5}$. Si bien, desde los espacios de decisión política se ha intentado dar respuestas a algunos de los problemas (GES, Ricarte Soto, entre otros), como sociedad, hasta ahora no hemos sido capaces de generar los acuerdos que permitan impulsar los cambios estructurales en salud que nuestro sistema necesita.

Un elemento significativamente diferente que ha ocurrido desde el 18 de octubre de 2019, es el grado de conciencia colectiva respecto de la necesidad de hacer cambios estructurales a nuestro sistema de salud. En este nuevo escenario, el espectro de cambios es amplio, abarcando desde la inserción de salud en la nueva constitución política hasta las maneras de financiar y de organizar nuestro sistema de salud.

Respecto de lo primero, la concepción del rol subsidiario del Estado plasmada en la Constitución Política de 1980, por una parte, se expresa una definición de la salud como derecho individual expresado en el numeral nueve del artículo 19 al establecer que "El Estado protege el libre e igualitario acceso a las acciones de promoción, protección y recuperación de la salud y de rehabilitación del individuo" ${ }^{6}$ (Constitución Política de Chile:18). Por otra parte, el inciso final "Cada persona tendrá el derecho a elegir el sistema de salud al que desee acogerse, sea éste estatal o privado señala" ${ }^{6}$, más allá de las interpretaciones jurídico-constitucionales 7,8 (Jordán, 2013; Nancuante et al., 2012) REF), en la práctica ha sido un factor que ha obstaculizado avanzar hacia cambios estructurales del sistema que fortalezcan la cohesión social. En el contexto de una nueva Constitución, por lo tanto, lo esperable es que salud sea efectivamente reconocido como derecho social y que el Estado debe garantizar.

Al mirar desde una perspectiva sistémica, los desafíos que como sociedad debemos enfrentar respecto a nuestro sistema de salud se pueden agrupar en tres grandes ámbitos: i) reformas que debemos hacer en el componente de financiamiento para que tengamos un verdadero sistema de seguridad social en salud para toda la población; ii) reformas orientadas a fortalecer y modernizar el sistema prestador público de atenciones de salud, que es el sistema principal que como país tenemos; iii) políticas y estrategias de desarrollo de recursos humanos.

Las áreas antes mencionadas están, obviamente, imbricadas. Debemos recordar que el componente de financiamiento es un mecanismo mediante el cual las perso- 
nas acceden a la atención de salud, la que es entregada por las personas que trabajan en los diferentes tipos de establecimientos de salud que el país tiene a lo largo de su territorio. En este sentido, es razonable que como sociedad enfrentemos estos desafíos desde una mirada global y compartida respecto del sistema de salud que queremos, de manera que los cambios que impulsemos en cada componente específico del sistema se orienten en la misma dirección.

Las reflexiones que se desarrollan en este documento, sin perder esa perspectiva global recién mencionada, se centran en el componente del financiamiento de nuestro sistema de salud. Lo que se busca es entregar elementos que contribuyan a un debate informado en el que participe toda la ciudadanía.

Las propuestas que se han conocido públicamente respecto de financiamiento del sistema de salud se pueden sintetizar en dos grandes modalidades: Financiamiento en base a impuestos generales y $\mathrm{Fi}$ nanciamiento en base a contribuciones vinculadas al trabajo. Respecto de la segunda propuesta, se han planteado las alternativas de Seguro Nacional de Salud $^{4,9}$ (ESP-COLMED, 2018; (CIPER, 2019) y de Multiseguros ${ }^{10}$ (Paolucci y Velasco, 2017).

En rigor, la primera propuesta se ha presentado como Sistema Único de Salud, pero de manera específica plantea el componente de financiamiento sobre la base de impuestos generales, además de un sistema prestador de atención de salud con prestadores estatales organizados en un Servicio Nacional de Salud. Así planteado, esta concepción se ajusta de manera estricta al Modelo Beveridge, creado en el Reino Unido en $1948^{11}$. Cabe hacer esta aclaración, pues la noción de Sistema Único de Salud es genérica, en tanto las otras propuestas se refieren de manera específica al componente de financiamiento. Más aún, algunos de los autores de propuestas de financiamiento, también han hecho propuestas específicas para otros componentes del sistema de salud, como el sistema prestador público ${ }^{5}$ (Arteaga y col., 2017).

En términos generales, lo que mejor distingue a las alternativas de financiamiento de los sistemas de salud es cómo se distribuye la carga de contribución al financiamiento en el total de la población. De este modo, los sistemas que descansan en impuestos generales, incluyen la carga del financiamien- to de la salud en el sistema tributario general. Esto permite que quienes tienen mayores ingresos y que por ello tributan más, contribuyan más al financiamiento que quienes tienen menos ingresos o simplemente no tienen ingresos. En el otro extremo, los sistemas que descansan en altos gastos de bolsillo derivados de co-pagos o pagos de seguros privados son proporcionales al uso de servicios de atención de salud y no a ingresos, lo que implica un peso proporcionalmente mayor del financiamiento en población de menores ingresos ${ }^{12}$.

¿Cuáles son, entonces, las ventajas y desventajas de las modalidades de financiamiento que se plantean en el actual escenario?

Lo que se desprende de la demanda ciudadana de cambios estructurales en salud es que, como sociedad, debemos abordar el desafío de avanzar hacia un verdadero arreglo de Seguridad Social. En este sentido, todas las modalidades que se analizan a continuación corresponden a sistemas de seguridad social en salud.

La modalidad de impuestos generales tiene como principal ventaja la progresividad de su financiamiento, lo que significa que las personas con mayores ingresos y que pagan más impuestos contribuyen más al financiamiento del sistema. Sin embargo, esta ventaja dependerá de la estructura tributaria del país. En Chile, 42\% de los ingresos que el fisco recauda corresponde a IVA, mientras que el promedio de los países de la Organización para la Cooperación y Desarrollo Económicos es de solo $20 \%{ }^{13}$. El IVA es un impuesto regresivo, pues como se agrega al precio de un bien o servicio, dicho monto es el mismo para todas las personas que compran ese bien o servicio; de este modo, el mayor esfuerzo de pago recae en las personas con ingresos menores. En el mismo sentido, mientras mayor sea el peso relativo del IVA en los ingresos fiscales que financian el presupuesto nacional, menos progresivo en el financiamiento.

Entre los aspectos positivos del financiamiento en base a impuestos generales se reconoce que en este tipo de sistema se generaría un mayor control del público sobre el gasto en salud, tanto en las crisis de corto plazo y cuando las prioridades de largo plazo se modifican ${ }^{12}$. De esta manera, el financiamiento estaría más protegido frente a los ciclos económicos y sus efectos en el mercado del trabajo. Complementariamente, existiría un mejor control de la 
inflación de costos en comparación con otros sistemas. La literatura referida a los sistemas con esta modalidad plantea que con frecuencia existirían niveles de sub-financiamiento que pueden conducir a racionamiento de prestaciones necesarias y deterioro de la calidad de los servicios. De igual modo, se restringe de manera importante la libertad de elección, lo que, a su vez, se asocia con menores niveles de satisfacción usuaria ${ }^{11}$, pudiendo también llegar a tener importantes implicancias en los niveles de equidad ${ }^{14}$ (Goodyear-Smith y Ashton, 2019). Lo anterior puede incentivar el desarrollo de seguros privados para reducir las dificultades de acceso a la atención de salud.

Los sistemas con financiamiento en base a contribuciones vinculadas al trabajo, es decir, cotizaciones obligatorias para salud de trabajadores, empleadores y del Estado, pueden organizarse como Seguro Nacional de Salud y como Multiseguros. En el primero, existe una sola entidad aseguradora de toda la población, en tanto en el sistema de multiseguros, existe un número variable de entidades que aseguran a la población que tienen a cargo.

En el Seguro Nacional de Salud ${ }^{15}$, el aseguramiento descansa en la distribución de los riesgos en el conjunto de la población, a la vez que se solidariza el financiamiento mediante la obligatoriedad de todas las personas de contribuir al financiamiento en función de su nivel de ingresos, lo que permite constituir un Fondo Único Nacional. La provisión de servicios de salud puede ser pública o privada, con o sin fines de lucro, considerándose la posibilidad de elegir prestadores. También pueden existir seguros complementarios voluntarios.

$\mathrm{Al}$ igual que la modalidad de financiamiento en base a impuestos generales, esta modalidad tiene ventajas asociadas con equidad y de efectividad sanitaria. Los mecanismos de transferencia de recursos son definidos por el Seguro en función de lograr eficacia sanitaria y eficiencia y, como es una única entidad, la que transfiere recursos a los prestadores (compra de prestaciones), los costos de transacción asociados a procesos administrativos y regulatorios son bajos, lo que constituye otra importante venta$\mathrm{ja}^{16}$.

El pago a prestadores puede hacerse directamente desde la entidad aseguradora a prestadores que entregan los servicios a la población o a gestores de redes con población a cargo, en cuyo caso los recursos se transfieren mediante fórmula de distribución (ej. per cápita corregido por necesidad sanitaria), para que estos gestores usen dichos recursos financieros para articular a los prestadores de su red.

La principal desventaja que se reconoce al Sistema de Seguro Nacional de Salud es el riesgo de captura de los niveles de decisión por parte de grupos de interés. Si la relación entre el Seguro Nacional y los prestadores está mediada por gestores de red este riesgo se puede atenuar, pues existiría una inteligencia sanitaria que, con los recursos que se le asignan, modula la compra de prestaciones a su red de prestadores con un enfoque de salud pública para satisfacer las necesidades de la población que tiene a cargo.

En la modalidad de Multiseguro existen varia entidades que participan del aseguramiento ${ }^{17,18}$. Estas entidades son públicas o privadas, normalmente sin fines de lucro. Al igual que todas las modalidades previamente analizadas, el aseguramiento es universal. Esto significa que las entidades aseguradoras no pueden discriminar por riesgo individual de las personas, lo que implica que deben existir mecanismos de ajustes para compensar los riesgos de salud asociados a las carteras de beneficiarios de cada entidad aseguradora. Las prestaciones de salud son provistas por prestadores públicos o privado en convenio con las entidades aseguradoras, siendo la mayoría prestadores privados en los países europeos.

Las ventajas que se asociarían con esta modalidad se relacionarían con la competencia regulada entre las entidades aseguradoras, lo que redundaría en eficiencia y satisfacción de los usuarios ${ }^{19}$. Las desventajas más relevantes se asocian con tres ámbitos de problemas. Por una parte, si bien existen mecanismos de compensación de riesgos que tienen por propósito evitar la discriminación de las personas debido a sus riesgos individuales por parte de las aseguradoras, estos mecanismos resultan insuficientes y en la literatura se reconoce la mantención de riesgos de segmentación ${ }^{20}$. Por otra parte, asociado con el punto anterior, se requiere una fuerte institucionalidad reguladora, lo que se traduce en mayores costos de transacción, los que pueden llegar a ser muy significativos ${ }^{16}$. Finalmente, dado que la unidad poblacional corresponde a la cartera de cada entidad aseguradora, se pierde la planificación sanitaria basada en poblaciones que habitan un territorio, afectándose el valor sanitario asociado al desa- 
rrollo de la estrategia de atención primaria.

Los arreglos basados en contribuciones vinculadas al trabajo (Seguro Único Nacional, Multiseguros), están más expuestos a las variaciones del mercado del trabajo y un desafío particular es cómo lograr que los trabajadores informales contribuyan al financiamiento del sistema. Desde una perspectiva del gasto en salud, una mayor aceptabilidad social y política al incremento de primas que al incremento de impuestos, expone al sistema de salud a mayores presiones sobre el gasto en salud que un sistema de financiamiento basado en impuestos generales.

El estallido social de una sociedad que reclama recuperar el protagonismo en la construcción de políticas públicas ha abierto una nueva oportunidad para que impulsemos los cambios estructurales que requerimos en salud.

El desafío mayor es que los cambios que impulsemos nos ayuden a fortalecer la cohesión social. En este sentido, es importante reconocer dos niveles de jerarquía en las decisiones que debemos adoptar. La decisión de mayor jerarquía y que implica un cambio fundamental para terminar con la segmentación social y discriminación en nuestro sistema de salud es que podamos mancomunar todos los recursos destinados a salud. Dada la estructura de nuestro sistema tributario, incluso con las propuestas de cambio que se plantean, el cambiar hacia una modalidad de sistema de salud basado en impuestos generales pierde su principal fundamento, que es el de la justicia social derivada de la progresividad del financiamiento. Sin embargo, esta decisión de mayor jerarquía, es decir el objetivo de mancomunar, aún se puede alcanzar si conformamos un Fondo Nacional de Salud.

En otro nivel de jerarquía decisional, que es secundario a la decisión que como país adoptemos respecto de un conformar un Fondo Nacional de Salud, podremos discutir el arreglo específico. En este nivel se abren las opciones de tener una sola entidad aseguradora que administre el Fondo (Seguro Nacional de Salud) o tener varias entidades aseguradoras participantes (Multiseguros). Cada una de estas opciones tiene ventajas y desventajas. Como sociedad, en forma democrática, tendremos que decidir cuál es aquella por la que optamos. Lo más importante es que para llegar a hacer esa discusión después de la decisión de primera jerarquía antes señalada, ya habremos resuelto que estamos en el terreno de la seguridad social habiendo dejado atrás un sistema que segrega a la población en función de los riesgos sanitarios y del nivel de ingreso de las personas, habremos dejado atrás un sistema que institucionaliza las inequidades para construir otro que efectivamente contribuye a la cohesión social que como país necesitamos para avanzar hacia el desarrollo.

Finalmente, es importante tener en mente que un cambio en la modalidad de financiamiento de nuestro sistema de salud, requiere que los recursos financieros para el sistema se incrementen, lo que se significa llegar al nivel de gasto público de $6 \%$ que propone OMS, además de impulsar una reforma del sistema prestador público para modernizarlo y fortalecerlo.

\section{REFERENCIAS BIBLIOGRÁFICAS}

1. Arteaga, O, Astorga, I., Pinto, A.M. (2002); “Desigualdades en la provisión de asistencia médica en el sector público de salud en Chile". Cadernos de Saúde Publica, 2002; 18(4): 1053-1066.

2. Albala, C., Sánchez, H., Lera, L., Angel, B. y Cea, X..Efecto sobre la salud de las desigualdades socioeconómicas en el adulto mayor. Resultados basales del estudio expectativa de vida saludable y discapacidad relacionada con la obesidad (Alexandros). Rev Med Chile 2011; 139: 1276-1285.

3. Jiménez, S., Barriga, O. y Salazar, A. Inequidad en el acceso a salud en Chile: Estudio multifactorial basado en la encuesta CASEN del año 2013. Rev Chil Salud Pública 2018; Vol 22 (1): 31-40.

4. ESP-COLMED. Propuestas para una reforma integral al financiamiento de la salud en Chile. Escuela de Salud Pública de la Universidad de Chile, Colegio Médico de Chile. Disponible en: file://C:/Users/Usuario/ Downloads/lee\%20aqui\%20la\%20propuesta\%20 lanzada\%20por\%20esp\%20y\%20colmed\%20(1). pdf. 2018.

5. Arteaga, O., Bayer, H., Martínez, S., Santelices, E., Velasco, C., Villarino, S. (2017). Propuesta de modernización y fortalecimiento de los prestadores estatales de servicios de salud. Publicación Escuela de Salud Pública $U$ de Chile y Centro de Estudios Públicos (CEP). Santiago de Chile. 2017.

6. Constitución Política de Chile. Texto promulgado por Decreto Supremo N ${ }^{\circ} 91.150$ del Ministerio del Interior de 21 de octubre de 1980. Editorial Jurídica de Chile: Santiago de Chile. 1981 
7. Jordán, T. (2013): “El cambio del eje referenciador del derecho a la protección de la salud a partir de la jurisprudencia constitucional sobre el subsistema privado de salud", en Estudios Constitucionales, 2013 (año 11 No 1) pp. 333-380.

8. Nancuante, U., Romer o, A., Sotomayor, R.: Régimen jurídico de la salud (Santiago, Legal Publishing Chile). 2012.

9. CIPER. Por un Nuevo Pacto Social en Salud para Chile. Disponible en https://ciperchile.cl/2019/11/30/porun-nuevo-pacto-social-en-salud-para-chile/. 2019.

10. Paolucci, F., Velasco, C. Reformando el sistema de seguros de salud chileno: Elección, competencia regulada y subsidios por riesgo. Centro de Estudio Públicos. CEP: Santiago de Chile. Disponible en: file:///C:/Users/ Usuario/Dropbox/Escuela\%20Sal\%20Pub/Temas/ Reforma/CEP\%20Isapres/dpp_025_septiembre2017_ paolucci_velasco.pdf. 2017.

11. European Observatory. United Kingdom: Country Health Profile. Disponible en: 2019http://www.euro. who.int/_data/assets/pdf_file/0006/419478/Country-Health-Profile-2019-United-Kingdom.pdf?ua =1. 2019.

12. Mossialos, E., Dixxon, A., Figueras, J., Kutzin, J. Funding health care: Options for Europe. Eupean Observatory on Health Care Systems Series. Open University Press: Buckingham-Philadelphia. 2002.

13. OECD. Revenue Statistics 2019 - Chile. Disponible en: https://www.oecd.org/tax/revenue-statistics-chile. pdf. 2019.

14. Goodyear-Smith, F., Ashton, T. New Zealand health system: universalism struggles with persisting inequities. Lancet 2019; 394: 432-42.
15. Martin, D., Miller, A., Quesnel-Vallée, A., Caron, N., Vissandjée, B., Marchildon, G. Canada's universal health-care system: achieving its potential. Lancet 2018; 391: 1718-35.

16. Morra, D., Nicholson, S., Levinson, W., Gans, D., Hammons, T., Casalino, L. US Physician Practices Versus Canadians: Spending Nearly Four Times As Much Money Interacting With Payers. Health Affairs 30, NO. 8 (2011): 1443-1450

17. European Observatory. Germany: Country Health Profile. Disponible en: http://www.euro.who.int/_data/ assets/pdf_file/0005/419459/Country-Health-Profile-2019-Germany.pdf?ua=1. 2019.

18. European Observatory. Belgium: Country Health Profile. Disponible en: http://www.euro.who.int/_data/ assets/pdf_file/0006/419451/Country-Health-Profile-2019-Belgium.pdf?ua=1. 2019.

19. European Observatory (2019d). The Netherlands: Country Health Profile. Disponible en: http://www. euro.who.int/_data/assets/pdf_file/0005/419477/ Country-Health-Profile-2019-the-Netherlands.pdf?ua=1. 2019.

20. Roman-Urrestarazu, A., Yang, J., Ettelt, S., Thalmann, I., Seguel Ravest, V., Brayne, C. Private health insurance in Germany and Chile: two stories of co-existence, segmentation and conflict. International Journal for Equity in Health (2018) 17:112 https://doi. org/10.1186/s12939-018-0831-z. 